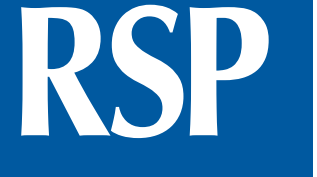

http://www.rsp.fsp.usp.br/

Revista de

Saúde Pública

\title{
Deficiência de glicose-6-fosfato desidrogenase e uso de primaquina: estimativa de custos de profissionais por macrocusteio e microcusteio
}

\author{
Henry Maia Peixoto ${ }^{1,11}$, Marcelo Augusto Mota Brito ${ }^{\text {III,IV }}$, Gustavo Adolfo Sierra Romero, ${ }^{1,11}$, Wuelton \\ Marcelo Monteiro ${ }^{\mathrm{III}, \mathrm{VV}}$, Marcus Vinícius Guimarães de Lacerda ${ }^{\mathrm{II}, \mathrm{V}}$, Maria Regina Fernandes de Oliveira ${ }^{\mathrm{l}, \mathrm{II}}$ \\ I Núcleo de Medicina Tropical. Universidade de Brasília. Brasília, DF, Brasil \\ " Instituto de Avaliação de Tecnologia em Saúde. Porto Alegre, RS, Brasil \\ III Fundação de Medicina Tropical Dr. Heitor Vieira Dourado. Manaus, AM, Brasil \\ Iv Universidade Estadual do Amazonas. Manaus, AM, Brasil \\ $\checkmark$ Instituto Leônidas e Maria Deane. Fundação Oswaldo Cruz. Manaus, AM, Brasil
}

\section{RESUMO}

A pesquisa teve por objetivo estudar se o macrocusteio, baseado no valor médio identificado no Sistema de Internação Hospitalar (SIH/SUS), constitui um bom estimador do custo de profissionais de saúde por paciente, tendo como comparação o método de microcusteio. O estudo foi desenvolvido no contexto da assistência hospitalar oferecida ao portador da deficiência de glicose-6-fosfato desidrogenase (dG6PD) do sexo masculino com evento adverso grave devido ao uso da primaquina, na Amazônia Brasileira.

O macrocusteio baseado no gasto em serviços profissionais do SIH/SUS, como proxy desse custo, correspondeu a $\mathrm{R} \$ 60,71$, e o microcusteio, baseado nos salários do médico $(\mathrm{R} \$ 30,43)$, do enfermeiro ( $R \$ 16,33$ ) e do técnico de enfermagem $(R \$ 5,93)$, estimou um custo total de $R \$ 52,68$. A diferença foi de apenas $\mathrm{R} \$ 8,03$, mostrando que os valores pagos pela Autorização de Internação Hospitalar (AIH) são estimadores próximos daqueles obtidos por técnica de microcusteio para os profissionais envolvidos diretamente no cuidado.

DESCRITORES: Deficiência de glicose-6-fosfato desidrogenase, economia. Primaquina, efeitos adversos. Malária, prevenção \& controle. Custos de Cuidados de Saúde. Sistema Único de Saúde, economia.

Recebido: 8 abr 2016

Aprovado: 8 dez 2016

Como citar: Peixoto HM,

Brito MAM, Romero GAS,

Monteiro WM, Lacerda MVG,

Oliveira MRF. Deficiência de glicose-6-fosfato desidrogenase e uso de primaquina: estimativa de custos de profissionais por macrocusteio e microcusteio. Rev Saude Publica. 2017:51:90.

Copyright: Este é um artigo de acesso aberto distribuído sob os termos da Licença de Atribuição Creative Commons, que permite uso irrestrito, distribuição e reprodução em qualquer meio desde que o autor e a fonte originais sejam creditados. 


\section{INTRODUÇÃO}

A primaquina, uma 8-aminoquinolina, é atualmente a única droga licenciada no Brasil para o tratamento radical da malária pelo Plasmodium vivax. Contribui para evitar as recaídas nesse tipo de malária e, consequentemente, diminui o número de portadores desse parasito. Porém, essa medicação vem sendo associada à hemólise grave, à internação e até mesmo ao óbito de indivíduos do sexo masculino portadores da deficiência da enzima glicose-6-fosfato desidrogenase (dG6PD), constituindo a principal ameaça aos portadores da dG6PD no Brasil ${ }^{1,2}$. Na Amazônia Brasileira, a prevalência estimada da dG6PD é 4,5\% ${ }^{3}$. Estimativas apresentadas por Peixoto et al. ${ }^{2}$ indicam que a dG6PD no âmbito da malária é onerosa para o Sistema Único de Saúde(SUS), especialmente em decorrência dos custos das hospitalizações dos pacientes que apresentam eventos adversos graves associados ao uso da primaquina. Nesse contexto, a análise de custo-efetividade apresentada por Peixoto et al. ${ }^{4}$ mostra que o uso do teste rápido CareStart ${ }^{\mathrm{TM}} \mathrm{G} 6 \mathrm{PD}$ antes da administração da primaquina é eficiente para diagnosticar a dG6PD e evitar a hospitalização na Amazônia Brasileira.

A validade das avaliações econômicas depende, em grande parte, do método e da precisão com que os custos foram avaliados. Os custos podem ser obtidos a partir do detalhamento dos itens e dos recursos utilizados (microcusteio), por meio de estimativas globais (macrocusteio) ou por abordagens mistas que utilizam tanto o macrocusteio quanto o microcusteio. Uma abordagem baseada no macrocusteio pode ser mais adequada quando se pretende representar a variabilidade de uma determinada região, ampliando a validade externa da avaliação; já o microcusteio reflete as características de uma amostra, o que limita a generalização, apesar de ser mais preciso ${ }^{6}$.

No Brasil, o Sistema de Internação Hospitalar (SIH/SUS) disponibiliza os valores das internações pagos pelo SUS às instituições de saúde, subsidiando a obtenção dos custos globais de acordo com a causa da internação e contemplando os serviços hospitalares e profissionais. Em decorrência do debate sobre o subfinanciamento relacionado aos valores pagos pelo SUS, o desenvolvimento de metodologias de microcusteio com o objetivo de estimar os custos hospitalares poderia contribuir com análises econômicas mais precisas.

O presente estudo teve como objetivo identificar se o macrocusteio dos serviços profissionais, baseado no valor médio pago por esses serviços, identificado no SIH/SUS, constitui um bom estimador do custo de profissionais de saúde, tendo como referência o microcusteio baseado nos salários do médico, do enfermeiro e do técnico de enfermagem no contexto da assistência hospitalar prestada ao portador da dG6PD do sexo masculino com evento adverso grave devido ao uso da primaquina na Amazônia Brasileira.

\section{MÉTODOS}

Foi realizada uma comparação de métodos de custeio no contexto de análise econômica desenvolvida na perspectiva do SUS para o ano de 2013, considerando todos os nove Estados da Amazônia Brasileira. O custo do serviço profissional com a hospitalização de portador da dG6PD submetido ao tratamento radical da infecção por $P$. vivax com primaquina foi obtido por meio de duas técnicas: a de macrocusteio (gross-costing ou top-down), realizado a partir de um banco de dados administrativo, baseado no componente Serviço Profissional da Autorização de Internação Hospitalar (AIH) pago pelo SUS; e a de microcusteio (microcosting ou bottom-up), fundamentada na casuística da Fundação de Medicina Tropical Dr. Heitor Vieira Dourado (FMT-HVD), centro de referência na atenção e pesquisa em malária no Estado do Amazonas, integralmente financiado pelo SUS, localizada em Manaus.

O macrocusteio obteve o valor médio do componente Serviço Profissional da AIH por meio da análise dos bancos de dados do SIH/SUS, utilizando-se o seguinte procedimento: os 108 arquivos que contêm os dados de registro do SIH/SUS dos nove Estados da Amazônia Brasileira, correspondentes ao ano de 2013, foram obtidos no formato *.dbf e exportados para o software Statistical Package for the Social Sciences (SPSS ${ }^{\circ}$, versão 20.0, transformando-os no formato *.sav; os bancos foram analisados individualmente no SPSS . Por meio do uso da função 
localizar do SPSS , foram identificados os indivíduos do sexo masculino maiores de seis meses hospitalizados para o tratamento de anemia hemolítica em decorrência da dG6PD (CID10 D550). Posteriormente, os resultados das análises foram agrupados em um único arquivo, possibilitando a obtenção do valor médio do referido componente. Embora o componente serviço profissional da AIH refira-se principalmente aos custos advindos do serviço do médico, o referido componente também foi utilizado como proxy dos custos profissionais totais (médico, enfermeiro e técnico de enfermagem), uma vez que não é possível discriminar, do componente serviço hospitalar da AIH, o custo do serviço dos demais profissionais.

O microcusteio foi baseado nos salários médios, praticados pelo Estado do Amazonas no ano de 2013, do médico, do enfermeiro e do técnico de enfermagem (custo profissional total), viabilizando a comparação entre o custo profissional total, o custo com o profissional médico e o valor obtido a partir da análise do componente serviço profissional da AIH. A partir do salário mensal, foi identificado o valor de uma hora de trabalho (salário mensal dividido pela carga horária mensal), seguida por sua multiplicação pela estimativa de horas dedicadas ao paciente, que foi obtida por meio da multiplicação entre média de dias de internação (4,58 dias) e 24 horas. Em seguida, esse produto foi ponderado pela proporção de pacientes internados por dG6PD na FMT-HVD, uma vez que os profissionais de saúde não cuidam exclusivamente dos pacientes internados por dG6PD (Tabela). A média de dias internados e o número de pacientes internados por dG6PD que subsidiaram o cálculo da proporção de internados foram obtidas a partir de informações não publicadas advindas do acompanhamento de 33 portadores da dG6PD do sexo masculino, com idade média de 24,3 anos, internados na FMT-HVD entre os anos 2009 e 2011, devido a eventos adversos da primaquina. Assim, a proporção de internados por dG6PD foi calculada a partir da divisão entre as 33 internações de portadores da deficiência enzimática e as 8.654 internações por todas as causas na instituição ocorridas no mesmo período (totalizando $0,38 \%$ ).

Foram realizadas análises de sensibilidade univariadas baseadas nos intervalos de $95 \%$ de confiança (IC95\%) dos seguintes parâmetros: proporção de internação por dG6PD e média de dias de internação.

\section{RESULTADOS}

O custo médio dos serviços profissionais obtido pela técnica de macrocusteio, baseada na análise das internações identificadas no SIH/SUS, correspondeu a R\$60,71. O microcusteio, baseado nos salários do médico, do enfermeiro e do técnico de enfermagem, indicou um custo de $\mathrm{R} \$ 52,68$, com preponderância do custo com o profissional médico (R $\$ 30,43)$, seguido

Tabela. Estimativa do custo dos serviços profissionais envolvidos na hospitalização de um portador da dG6PD infectado pelo P. vivax após uso de primaquina na Amazônia Brasileira, 2013.

\begin{tabular}{|c|c|c|c|c|c|c|c|}
\hline \multirow{3}{*}{ Profissionais } & \multirow{3}{*}{$\begin{array}{l}\text { Salário Mensal } \\
\text { (R\$) }\end{array}$} & (B) & \multirow{3}{*}{$\begin{array}{c}\frac{(C)}{\begin{array}{c}\text { Carga horária/ } \\
\text { mês (horas) }\end{array}} \\
C=B^{* 4}\end{array}$} & \multirow{3}{*}{$\begin{array}{c}\text { (D) } \\
\begin{array}{c}\text { Valor da hora } \\
\text { trabalhada }\end{array} \\
\text { (A/C) }\end{array}$} & \multirow{3}{*}{$\begin{array}{c}\frac{(E 1)}{c} \\
\begin{array}{c}\text { Custo profissional por } \\
\text { paciente }\end{array} \\
E=D^{*} 4,58^{\mathrm{a} *} 24 \mathrm{~h}^{*} 0,38 \%{ }^{\mathrm{b}}\end{array}$} & \multicolumn{2}{|c|}{ Análise de sensibilidade } \\
\hline & & \multirow{2}{*}{$\begin{array}{l}\text { Carga horária/ } \\
\text { semana (horas) }\end{array}$} & & & & \multirow{2}{*}{$\begin{array}{c}\text { (E2) } \\
\begin{array}{c}\text { Proporção de } \\
\text { internados } \\
(0,25 \%-0,51 \%)^{\mathrm{c}}\end{array}\end{array}$} & \multirow{2}{*}{$\begin{array}{c}\text { (E3) } \\
\text { Média de dias } \\
\text { de internação } \\
(2,58-6,57)^{d}\end{array}$} \\
\hline & & & & & & & \\
\hline Médico & $5.832,98$ & 20 & 80 & 72,91 & 30,43 & $20,02-40,84$ & $17,16-43,69$ \\
\hline Enfermeiro & $4.694,41$ & 30 & 120 & 39,12 & 16,33 & $10,74-21,91$ & $9,20-23,44$ \\
\hline $\begin{array}{l}\text { Técnico de } \\
\text { enfermagem }\end{array}$ & $1.704,1$ & 30 & 120 & 14,21 & 5,93 & $3,90-7,95$ & $3,34-8,51$ \\
\hline Total & & & & & 52,68 & $34,66-70,70$ & $29,70-75,64$ \\
\hline \multirow{2}{*}{\multicolumn{4}{|c|}{ Diferença entre custos: macrocusteio versus microcusteio }} & Diferença $1^{\mathrm{e}}$ & 30,28 & $19,87-40,69$ & $17,02-43,55$ \\
\hline & & & & Diferença $2^{f}$ & 8,03 & $-9,99-26,05$ & $-14,93-31,01$ \\
\hline
\end{tabular}

${ }^{a}$ Média de dias de internação por dG6PD na FMT-HVD.

b Proporção de internação por dG6PD entre os internados na FMT-HVD.

' Variação do custo profissional por paciente baseada no intervalo de confiança (IC95\%) da proporção de internados por dG6PD entre os internados na FMT-HVD.

¿ Variação do custo profissional por paciente baseada no intervalo de confiança (IC95\%) da média de dias de internação por dG6PD na FMT-HVD.

e Diferença entre macrocusteio $(R \$ 60,71)$ e microcusteio baseado apenas no custo do profissional médico.

${ }^{\mathrm{f}}$ Diferença entre macrocusteio $(\mathrm{R} \$ 60,71)$ e microcusteio baseado no custo profissional total (médico, enfermeiro e técnico de enfermagem). 
do enfermeiro ( $\mathrm{R} \$ 16,33)$ e do técnico de enfermagem $(\mathrm{R} \$ 5,93)$. Observa-se que a diferença entre os custos obtidos por meio das diferentes técnicas, considerando o custo profissional total, foi de apenas $\mathrm{R} \$ 8,03$ e considerando apenas os custos com o médico, de $\mathrm{R} \$ 30,28$ (Tabela). A análise de sensibilidade univariada realizada com a proporção de internação e a média de dias internados por dG6PD mostrou que o custo profissional total, obtido por meio do microcusteio, variou respectivamente: entre $R \$ 34,66$ e $R \$ 70,70$ e entre $R \$ 29,70$ e $\mathrm{R} \$ 75,64$ (Tabela).

\section{DISCUSSÃO}

Os custos hospitalares obtidos a partir da análise dos registros regionais e nacionais do SIH/SUS têm subsidiado diversas análises econômicas desenvolvidas na perspectiva do SUS, possibilitando, segundo Silva et al. ${ }^{7}$, uma visão mais abrangente. De acordo com a diretriz para avaliação econômica proposta pelo Ministério da Saúde (MS), os custos da internação obtidos a partir dos valores das AIH têm sido utilizados em quase todos os estudos realizados no Brasil, apesar de a AIH ser criticada por representar os valores reembolsados e não necessariamente os custos reais ${ }^{6}$. Nesse contexto, observa-se a utilização do valor médio da AIH obtidos a partir da análise do SIH/SUS, como valores proxy do custo, em estudos com os mais variados objetivos, como: avaliação de custos de doença ${ }^{2,8}$, análises da eficiência de vacinas $^{9,10}$, da eficiência de novas tecnologias diagnósticas ${ }^{4}$, da eficiência de programas ${ }^{11} \mathrm{e}$ da eficiência de novas tecnologias terapêuticas ${ }^{12}$, dentre outros.

A discussão sobre a desatualização dos valores pagos pela AIH também deve ser considerada nas análises econômicas. A esse respeito, Sampaio et al. ${ }^{13}$, ao estudarem a gestão financeira da neurocirurgia, compararam os valores pagos pelas AIH aos gastos catalogados por pacientes durante o período de agosto de 2012 a junho de 2013. Os autores ${ }^{13}$ identificaram um déficit de $\mathrm{R} \$ 395.329,17$ entre os valores obtidos por microcusteio ( $\mathrm{R} \$ 718.036,70)$ e o valor reembolsado pelas AIH $(\mathrm{R} \$ 321.607,45)$. Lucarevschi et al. ${ }^{14}$, ao combinarem as técnicas de microcusteio e macrocusteio para avaliar os custos hospitalares diretos relacionados à meningite pneumocócica em crianças, identificaram custos 10 a 20 vezes superiores aos pagos pela AIH. Dada à variedade de desfechos em saúde, é possível supor que o valor pago pela AIH também possa superestimar o custo da internação para alguns desfechos, embora o presente estudo não tenha identificado publicações que corroborem essa suposição.

No presente estudo, o valor dos serviços profissionais por microcusteio, considerando inicialmente apenas o custo do profissional médico, em comparação ao macrocusteio, apresentou valor correspondente à metade do valor reembolsado pela AIH. No entanto, quando considerados simultaneamente os custos do médico, do enfermeiro e do técnico de enfermagem, o valor obtido foi muito próximo ao valor do macrocusteio, indicando que a AIH pode ser uma boa proxy da estimativa de custos de serviços dos profissionais envolvidos diretamente no cuidado dos portadores de dG6PD quando comparada com a técnica de microcusteio.

Este estudo apresenta algumas limitações, como o fato de alguns parâmetros epidemiológicos e os custos utilizados no microcusteio procederem da casuística do Estado do Amazonas e serem extrapolados para toda a Amazônia Brasileira. As incertezas consideradas na análise de sensibilidade univariada indicaram que os custos profissionais não apresentaram variações expressivas, embora sofram a influência da variação da proporção de internado por dG6PD e dos dias de hospitalização.

A comparação entre o microcusteio e o macrocusteio é uma contribuição inédita para a discussão de técnicas de custeio para custos de profissionais em cenários de atenção à malária na Amazônia Brasileira, com potencial para subsidiar futuros estudos econômicos na área. Ressalta-se que a AIH é bastante utilizada no Brasil como fonte dos custos de assistência hospitalar, por facilitar a comparação entre estudos econômicos e representar os valores praticados na rotina dos serviços. 


\section{REFERÊNCIAS}

1. Monteiro WM, Franca GP, Melo GC, Queiroz ALM, Brito M, Peixoto HM, Oliveira MRF, et al. Clinical complications of G6PD deficiency in Latin American and Caribbean populations: systematic review and implications for malaria elimination programmes. Malar J. 2014;13:70. https://doi.org/10.1186/1475-2875-13-70

2. Peixoto HM, Brito MA, Romero GA, Monteiro WM, Lacerda MV, Oliveira MRF. G6PD deficiency in male individuals infected by Plasmodium vivax malaria in the Brazilian Amazon: a cost study. Malar J. 2015;14:126. https://doi.org/10.1186/s12936-015-0647-x

3. Santana MS, Monteiro WM, Siqueira AM, Costa MF, Sampaio V, Lacerda MV, et al. Glucose-6-phosphate dehydrogenase deficient variants are associated with reduced susceptibility to malaria in the Brazilian Amazon. Trans $\mathrm{R}$ Soc Trop Med Hyg. 2013;107(5):301-6. https://doi.org/10.1093/trstmh/trt015

4. Peixoto HM, Brito MAM, Romero GAS, Monteiro WM, Lacerda MVG, Oliveira MRF. Cost-effectiveness analysis of rapid diagnostic tests for G6PD deficiency in patients with Plasmodium vivax malaria in the Brazilian Amazon. Malar J. 2016;15:82. https://doi.org/10.1186/s12936-016-1140-x

5. Clement FM, Ghali WA, Donaldson CAM, Manns BJ. The impact of using different costing methods on the results of an economic evaluation of cardiac care : microcosting vs gross-costing approaches. Health Economics. 2009;18(4):377-88. https://doi.org/ 10.1002/hec.1363

6. Ministério da Saúde (BR). Diretrizes metodológicas: diretriz de avaliação econômica. 2.ed. Brasília (DF); 2014 [citado 27 jul 2017]. Disponível em: http://bvsms.saude.gov.br/bvs/ publicacoes/diretrizes_metodologicas_diretriz_avaliacao_economica.pdf

7. Silva EN, Silva MT, Pereira MG. Identificação, mensuração e valoração de custos em saúde. Epidemiol Serv Saude. 2016;25(2):437-9. https://doi.org/10.5123/S1679-49742016000200023

8. Bahia L, Coutinho ESF, Barufaldi LA, Abreu GA, Malhão TA, Souza CPR, et al. The costs of overweight and obesity-related diseases in the Brazilian public health system: cross-sectional study. BMC Public Health. 2012;12(1):440. https://doi.org/10.1186/1471-2458-12-440

9. Novaes HMD, Soárez PC, Silva GA, Ayres A, Itria A, Rama CH, et al. Cost-effectiveness analysis of introducing universal human papillomavirus vaccination of girls aged 11 years into the National Immunization Program in Brazil. Vaccine. 2015;33 Suppl 1:A135-42. https://doi.org/10.1016/j.vaccine.2014.12.031

10. Sartori AMC, Soárez PC, Fernandes EG, Gryninger LCF, Viscondi JYK, Novaes HMD. Cost-effectiveness analysis of universal maternal immunization with tetanus-diphtheria-acellular pertussis (Tdap) vaccine in Brazil. Vaccine. 2016;34(13):1531-9. https://doi.org/10.1016/j.vaccine.2016.02.026

11. Ribeiro RA, Caleffi M, CAP. Custo-efetividade de um programa de rastreamento organizado de câncer de mama no Sul do Bras. Cad Saude Publica. 2013;29 Supl 1:S131-45. https://doi.org/10.1590/0102-311X00005213

12. Ribeiro RA, Stella SF, Zimerman LI, Pimentel M, Rohde LE, Polanczyk CA. Custo-efetividade de cardiodesfibriladores implantáveis no Brasil nos setores público e privado. Arq Bras Cardiol. 2010;95(5):577-86. https://doi.org/10.1590/S0066-782X2010005000134

13. Sampaio GB, Moraes DF, Meguins LC, Adry RARC, Sampaio PVC. O custo da neurocirurgia no Sistema Único de Saúde no Hospital de Base de São José do Rio Preto. Arq Bras Neurocir. 2014 [citado 27 jul 2017];33(3):186-91. Disponível em: http://files.bvs.br/upload/S/0103-5355/2014/v33n3/756170.pdf

14. Lucarevschi BR, Escobar AMU, Grisi S. Custos hospitalares da meningite causada por Streptococcus pneumoniae na cidade de São José dos Campos, São Paulo, Brasil. Cad Saude Publica. 2012;28(4):740-8. https://doi.org/10.1590/S0102-311X2012000400013

Financiamento: Conselho Nacional de Desenvolvimento Científico e Tecnológico (CNPq - Chamada Universal MCTI/CNPq 14/2013) e Instituto de Avaliação de Tecnologia em Saúde (IATS - Chamada 20/2013).

Contribuição dos Autores: HMP e MRFO conceberam o estudo, redigiram o manuscrito e participaram da coleta, análise e interpretação dos dados. GASR, MVGL, WMM e MAMB conceberam o estudo, revisaram o manuscrito e participaram da coleta, analise e interpretação dos dados. Todos os autores aprovaram a versão final e assumem a responsabilidade pública pelo conteúdo do artigo.

Conflito de Interesses: Os autores declaram não haver conflito de interesses. 